\title{
Some Notes on $L_{p}$-metric Space of Fuzzy Sets
}

\author{
Yun Kyong Kim \\ Department of Information \& Communication Engineering, Dongshin University, Geonjaero 253, Naju, \\ Jeonnam, 520-714, Korea
}

\begin{abstract}
It is well-known that the space $E^{n}$ of fuzzy numbers (i.e., normal, upper-semicontinuous, compact-supported and convex fuzzy subsets) in the $n$-dimensional Euclidean space $R^{n}$ is separable but not complete with respect to the $L_{p}$-metric.

In this paper, we introduce the space $F_{p}\left(R^{n}\right)$ that is separable and complete with respect to the $L_{p}$-metric. This will be accomplished by assuming $p$-th mean bounded condition instead of compact-supported condition and by removing convex condition.
\end{abstract}

Key Words: Fuzzy numbers, Compact sets, $L_{p}$-metric.

\section{Introduction}

The metric in a space of fuzzy sets plays an important role both in the theory and in its applications. There are various useful metrics defined on the fuzzy number space $E^{n}$ of normal, upper-semicontinuous, compact-supported and convex fuzzy subsets of $n$-dimensional Euclidean space $R^{n}$. The readers may refer to [2] for supremum metric, sendograph metric and $L_{p}$-metric, and refer to [6] for Skorohod metric.

It is well-known that $E^{n}$ is complete and separable if it is equipped with the metric except $L_{p}$-metric. Characterizations of compact subsets of $E^{n}$ equipped supremum metric, sendograph metric and the Skorohod metric were given by Greco [4], Greco and Moschen [5], Greco [3], Zhao and $\mathrm{Wu}$ [10], Joo and Kim [6], respectively.

However, it is known that $E^{n}$ is separable but not complete with respect to the $L_{p}$-metric. This problem arises from the fact that compact-supported condition is inadequate for the $L_{p}$-metric.

Related to this problem, Kraschmer [7] dealt with completion of $E^{n}$ w.r.t. the $L_{p}$-metric by introducing the notion of support function for noncompact fuzzy number and Degang et al. [1] proposed the completion of $E^{1}$ w.r.t. the $L_{1}$-metric by using representation theorem of noncompact fuzzy number in $E^{1}$. But these approaches cannot be valid any more if we drop the convexity condition.

In this paper, we introduce the space $F_{p}\left(R^{n}\right)$ without convexity that is complete and separable with respect to the $L_{p}$-metric.

\section{Preliminaries}

Let $K\left(R^{n}\right)$ denote the family of all non-empty compact subsets of the $n$-dimensional Euclidean space $R^{n}$ with the usual norm $|\cdot|$. Then the space $K\left(R^{n}\right)$ is metrizable by the Hausdorff metric $h$ defined by

$$
h(A, B)=\max \left[\sup _{a \in A} \inf _{b \in B}|a-b|, \sup _{b \in B} \inf _{a \in A}|a-b|\right] .
$$

The norm of $A \in K\left(R^{n}\right)$ is defined by

$$
\|A\|=h(A,\{0\})=\sup _{a \in A}|a| .
$$

It is well-known that $K\left(R^{n}\right)$ is complete and separable with respect to the Hausdorff metric $h$. Also, if we denote by $K_{c}\left(R^{n}\right)$ the family of all $A \in K\left(R^{n}\right)$ which is convex, then $K_{c}\left(R^{n}\right)$ is a closed subspace of $\left(K\left(R^{n}\right), h\right)$.

Let $F\left(R^{n}\right)$ denote the family of all fuzzy sets $u: R^{n} \rightarrow$ $[0,1]$ with the following properties;

(i) $u$ is normal, i.e., there exists $x \in R^{n}$ such that $u(x)=$ 1 .

(ii) $L_{\alpha} u=\left\{x \in R^{n}: u(x) \geq \alpha\right\}$ is a compact subset of $R^{n}$ for each $0<\alpha \leq 1$.

$L_{\alpha} u$ is called the $\alpha$-level set of $u$. We denote by $F_{c}\left(R^{n}\right)$ the family of all $u \in F\left(R^{n}\right)$ which is convex, i.e., $u(\lambda x+(1-\lambda) y) \geq \min (u(x), u(y))$ for all $x, y \in R^{n}$ and $0 \leq \lambda \leq 1$. Then $u \in F_{c}\left(R^{n}\right)$ if and only if $L_{\alpha} u \in K_{c}\left(R^{n}\right)$ for each $0<\alpha \leq 1$.

Manuscript received May. 11, 2010; revised Aug. 19, 2010; 
Also, we denote by $F_{\infty}\left(R^{n}\right)$ (resp. $\left.F_{c \infty}\left(R^{n}\right)\right)$ the family of all $u \in F\left(R^{n}\right)$ (resp. $\left.F_{c}\left(R^{n}\right)\right)$ with compact support, i.e., $L_{0} u=\overline{\left\{x \in R^{n}: u(x)>0\right\}}$ is compact, where $\bar{A}$ denotes the closure of $A$ w.r.t. the usual norm in $R^{n}$. Briefly, $F_{c, \infty}\left(R^{n}\right)$ is denoted by $E^{n}$ and a member of $E^{n}$ is called a fuzzy number.

Joo and Kim [6] showed that $u \in F_{\infty}\left(R^{n}\right)$ can be characterized by a function $f_{u}$ defined as $f_{u}:[0,1] \rightarrow$ $K\left(R^{n}\right), f_{u}(\alpha)=L_{\alpha} u$, which is non-increasing, leftcontinuous on $(0,1]$, right-continuous at 0 and right-limits on $[0,1)$. By very similar arguments, we can obtain the following lemma.

Lemma 2.1. For $u \in F\left(R^{n}\right)$, we define

$$
f_{u}:(0,1] \longrightarrow\left(K\left(R^{n}\right), h\right), f_{u}(\alpha)=L_{\alpha} u .
$$

Then the followings hold;

(i) $f_{u}$ is non-increasing, i.e., $\alpha \leq \beta$ implies $f_{u}(\alpha) \supset$ $f_{u}(\beta)$,

(ii) $f_{u}$ is left continuous on $(0,1]$,

(iii) $f_{u}$ has right-limits on $(0,1)$.

Conversely, if $g:[0,1] \rightarrow K\left(R^{n}\right)$ is a function satisfying the above conditions $(i)-(i i i)$, then there exists a unique $v \in F\left(R^{n}\right)$ such that $g(\alpha)=L_{\alpha} v$ for all $\alpha \in(0,1]$.

If we denote by $L_{\alpha^{+}} u$ the right-limit of $f_{u}$ at $\alpha \in(0,1)$, then it is well-known that

$$
L_{\alpha^{+}} u=\overline{\left\{x \in R^{n}: u(x)>\alpha\right\}} .
$$

\section{Main Results}

The $L^{p}$-metric $d_{p}$ on the fuzzy number space $E^{n}$ is defined as follows;

$$
d_{p}(u, v)=\left(\int_{0}^{1} h\left(L_{\alpha} u, L_{\alpha} v\right)^{p} d \alpha\right)^{1 / p} .
$$

It is well-known that $\left(E^{n}, d_{p}\right)$ is separable but not complete. This fact seems to be natural since $E^{n}$ is too small for it to be complete w.r.t. $d_{p}$. In order to achieve completeness, we need to introduce a new family of fuzzy sets that includes $E^{n}$.

For $1 \leq p<\infty$, let $F_{p}\left(R^{n}\right)$ (resp. $\left.F_{c, p}\left(R^{n}\right)\right)$ be the family of all fuzzy sets $u \in F\left(R^{n}\right)$ (resp. $F_{c}\left(R^{n}\right)$ ) such that

$$
\int_{0}^{1}\left\|L_{\alpha} u\right\|^{p} d \alpha<\infty
$$

It is obvious that $F_{\infty}\left(R^{n}\right) \subset F_{p}\left(R^{n}\right)$ but $F_{\infty}\left(R^{n}\right) \neq$ $F_{p}\left(R^{n}\right)$. It is easy to prove that the $d_{p}$ on $F_{p}\left(R^{n}\right)$ satisfies the axioms of metric. We first prove the completeness of $\left(F_{p}\left(R^{n}\right), d_{p}\right)$

Theorem 3.1. $\left(F_{p}\left(R^{n}\right), d_{p}\right)$ is complete.

Proof. Let $\left\{u_{i}\right\}$ be a Cauchy sequence in $\left(F_{p}\left(R^{n}\right), d_{p}\right)$ such that $\int_{0}^{1} h\left(L_{\alpha} u_{i}, L_{\alpha} u_{j}\right)^{p} d \alpha \rightarrow 0$ as $i, j \rightarrow \infty$.

Step 1: First, we show that there exists a subsequence $\left\{u_{i_{k}}\right\}$ of $\left\{u_{i}\right\}$ such that $\left\{L_{\alpha} u_{i_{k}}\right\}$ is a Cauchy sequence in $\left(K\left(R^{n}\right), h\right)$ for almost all $\alpha$.

We note that for each $\varepsilon>0$,

$$
\begin{gathered}
\mu\left\{\alpha: h\left(L_{\alpha} u_{i}, L_{\alpha} u_{j}\right)>\varepsilon\right\} \\
\leq \frac{1}{\varepsilon^{p}} \int_{0}^{1} h\left(L_{\alpha} u_{i}, L_{\alpha} u_{j}\right)^{p} d \alpha \rightarrow 0
\end{gathered}
$$

as $i, j \rightarrow \infty$, where $\mu$ denote the Lebesgue measure.

For any positive integer $k$, we find an integer $N_{k}$ such that

$$
\mu\left(\left\{\alpha: h\left(L_{\alpha} u_{i}, L_{\alpha} u_{j}\right) \geq \frac{1}{2^{k}}\right\}\right)<\frac{1}{2^{k}}
$$

for $i, j \geq N_{k}$. Now we write

$$
i_{1}=N_{1}, i_{k}=\left(i_{k-1}+1\right) \vee N_{k} \text { for } \mathrm{k} \geq 2,
$$

then $\left\{u_{i_{k}}\right\}$ is a subsequence of $\left\{u_{i}\right\}$.

Let $I_{k}=\left\{\alpha: h\left(L_{\alpha} u_{i_{k}}, L_{\alpha} u_{i_{k+1}}\right) \geq \frac{1}{2^{k}}\right\}$ and

$$
I_{0}=\limsup _{k \rightarrow \infty} I_{k}=\cap_{m=1}^{\infty} \cup_{k=m}^{\infty} I_{k}
$$

Then since

$$
\mu\left(\cup_{k=m}^{\infty} I_{k}\right) \leq \sum_{k=m}^{\infty} \mu\left(I_{k}\right)<\frac{1}{2^{m-1}}
$$

we have that $\mu\left(I_{0}\right)=0$. And if $\alpha \notin I_{0}$, then there exists $m$ such that $\alpha \notin \cup_{k=m}^{\infty} I_{k}$ and so for $k, l \geq m$,

$$
h\left(L_{\alpha} u_{i_{k}}, L_{\alpha} u_{i_{l}}\right) \leq \sum_{k=m}^{\infty} h\left(L_{\alpha} u_{i_{k}}, L_{\alpha} u_{i_{k+1}}\right)<\frac{1}{2^{m-1}},
$$

which implies $\left\{L_{\alpha} u_{i_{k}}\right\}$ is a Cauchy sequence in $\left(K\left(R^{n}\right), h\right)$.

Step 2: By completeness of $\left(K\left(R^{n}\right), h\right),\left\{L_{\alpha} u_{i_{k}}\right\}$ converges to $A_{\alpha}$ for some $A_{\alpha} \in K\left(R^{n}\right)$ for each $\alpha \notin I_{0}$.

If $0<\alpha \leq 1$ and $\alpha \in I_{0}$, then we define

$$
A_{\alpha}=\cap_{\beta<\alpha, \beta \notin I_{0}} A_{\beta} .
$$

Then by Lemma 2.1, there exists a $u \in F\left(R^{n}\right)$ such that $L_{\alpha} u=A_{\alpha}$ for each $0<\alpha \leq 1$. Now we have to show that $u \in F_{p}\left(R^{n}\right)$ and $d_{p}\left(u_{i}, u\right) \rightarrow 0$ as $i \rightarrow \infty$. 
Since $\left\{u_{i_{k}}\right\}$ is Cauchy sequence in $\left(F_{p}\left(R^{n}\right), d_{p}\right)$, there exist an $M$ such that for $k, l \geq M$,

$$
\int_{0}^{1} h\left(L_{\alpha} u_{i_{k}}, L_{\alpha} u_{i_{l}}\right)^{p} d \alpha<1
$$

For a fixed $k \geq M$, since

$$
\lim _{l \rightarrow \infty} h\left(L_{\alpha} u_{i_{k}}, L_{\alpha} u_{i_{l}}\right)=h\left(L_{\alpha} u_{i_{k}}, L_{\alpha} u\right)
$$

for almost all $\alpha$, we have that by Fatou's lemma,

$$
\begin{aligned}
& \int_{0}^{1} h\left(L_{\alpha} u_{i_{k}}, L_{\alpha} u\right)^{p} d \alpha \\
\leq & \liminf _{l \rightarrow \infty} \int_{0}^{1} h\left(L_{\alpha} u_{i_{k}}, L_{\alpha} u_{i_{l}}\right)^{p} d \alpha \leq 1 .
\end{aligned}
$$

Thus,

$$
\begin{aligned}
& \int_{0}^{1}\left\|L_{\alpha} u\right\|^{p} d \alpha \\
\leq & 2^{p} \int_{0}^{1}\left\|L_{\alpha} u_{i_{k}}\right\|^{p} d \alpha+2^{p} \int_{0}^{1} h\left(L_{\alpha} u_{i_{k}}, L_{\alpha} u\right)^{p} d \alpha<\infty,
\end{aligned}
$$

which implies $u \in F_{p}\left(R^{n}\right)$.

Finally, the triangle inequality

$$
d_{p}\left(u_{i}, u\right) \leq d_{p}\left(u_{i}, u_{i_{k}}\right)+d_{p}\left(u_{i_{k}}, u\right)
$$

shows that $d_{p}\left(u_{i}, u\right) \rightarrow 0$ as $i \rightarrow \infty$.

Corollary 3.2. $\quad F_{c p}\left(R^{n}\right)$ is a closed subspace of $\left(F_{p}\left(R^{n}\right), d_{p}\right)$ and so it is complete.

Proof. Let $\left\{u_{i}\right\}$ be a sequence in $\left(F_{c p}\left(R^{n}\right), d_{p}\right)$ such that for some $v \in F_{p}\left(R^{n}\right)$,

$$
d_{p}\left(u_{i}, v\right) \rightarrow 0 \text { as } i \rightarrow \infty .
$$

Then there exists a $I \subset(0,1]$ with Lebesgue measure 0 such that for all $\alpha \notin I$,

$$
h\left(L_{\alpha} u_{i}, L_{\alpha} v\right) \rightarrow 0 \text { as } i \rightarrow \infty .
$$

Since $L_{\alpha} u_{i} \in K_{c}\left(R^{n}\right)$ and $K_{c}\left(R^{n}\right)$ is a closed subspace of $K\left(R^{n}\right), L_{\alpha} v \in K_{c}\left(R^{n}\right)$ for all $\alpha \notin I$. If $0<\alpha \leq 1$ and $\alpha \in I$, then we can choose a increasing sequence $\left\{\alpha_{k}\right\}$ with $\alpha_{k} \notin I$ so that $\alpha_{k} \rightarrow \alpha$ as $k \rightarrow \infty$. Then by left-continuity of $L_{\alpha} v$ as a function of $\alpha$, we have $h\left(L_{\alpha_{k}} v, L_{\alpha} v\right) \rightarrow 0$ as $k \rightarrow \infty$, and so $L_{\alpha} v \in K_{c}\left(R^{n}\right)$. This completes the proof.

Now we prove that $\left(F_{p}\left(R^{n}\right), d_{p}\right)$ is separable. To do this, we need some lemmas.

Lemma 3.3. If $A_{j}, B_{j} \in K\left(R^{n}\right), j=1,2$, then

$$
h\left(A_{1} \cup A_{2}, B_{1} \cup B_{2}\right) \leq \max \left[h\left(A_{1}, B_{1}\right), h\left(A_{2}, B_{2}\right)\right] .
$$

Proof. It follows from the fact that

$$
\begin{aligned}
& \sup _{a \in A_{1} \cup A_{2}} \inf _{b \in B_{1} \cup B_{2}}|a-b| \\
= & \max \left(\sup _{a \in A_{1}} \inf _{b \in B_{1} \cup B_{2}}|a-b|, \sup _{a \in A_{2}} \inf _{b \in B_{1} \cup B_{2}}|a-b|\right) \\
\leq & \max \left(\sup _{a \in A_{1}} \inf _{b \in B_{1}}|a-b|, \sup _{a \in A_{2}} \inf _{b \in B_{2}}|a-b|\right)
\end{aligned}
$$

Lemma 3.4. If $u \in F\left(R^{n}\right)$ and $0<\beta<1$, then there exists a partition $\beta=\beta_{0}<\cdots<\beta_{m}=1$ of $[\beta, 1]$ such that

$$
h\left(L_{\beta_{k}} u, L_{\beta_{k-1}^{+}} u\right)<\varepsilon \text { for all } k=1, \cdots, m .
$$

Proof. Let $\varepsilon>0$ be given. By applying Lemma 2.1, for each $\beta<\alpha<1$, we can take $\delta_{\alpha}>0$ so that

$$
h\left(L_{\alpha} u, L_{\alpha-\delta_{\alpha}} u\right)<\varepsilon
$$

and

$$
h\left(L_{\alpha^{+}} u, L_{\alpha+\delta_{\alpha}} u\right)<\varepsilon .
$$

Also, we can choose $\delta_{\beta}, \delta_{1}>0$ so that

$$
h\left(L_{\beta}+u, L_{\beta+\delta_{\beta}} u\right)<\varepsilon
$$

and

$$
h\left(L_{1} u, L_{1-\delta_{1}} u\right)<\varepsilon .
$$

Let $I_{\beta}=\left[\beta, \beta+\delta_{\beta}\right), I_{1}=\left(1-\delta_{1}, 1\right]$ and for each $\beta<$ $\alpha<1$,

$$
I_{\alpha}=\left(\alpha-\delta_{\alpha}, \alpha-\delta_{\alpha}\right) .
$$

Then by the compactness of $[\beta, 1]$, there exists $\alpha_{1}, \cdots, \alpha_{N} \in(\beta, 1)$ such that

$$
[0,1]=I_{\beta} \cup I_{1} \cup\left(\cup_{i=1}^{N} I_{\alpha_{i}}\right) .
$$

The collection of points $\left\{\beta, \beta+\delta_{\beta}, 1-\delta_{1}, 1\right\} \cup\left\{\alpha_{i}-\right.$ $\left.\delta_{\alpha_{i}}, \alpha_{i}, \alpha_{i}+\delta_{\alpha_{i}}: i=1, \cdots, N\right\}$ forms a partition of $[\beta, 1]$. We denote these points in ascending order by

$$
\beta=\beta_{0}<\beta_{1}<\cdots<\beta_{m}=1 .
$$

Then it is obvious that for all $k=1,2, \cdots, m$,

$$
h\left(L_{\beta_{k}} u, L_{\beta_{k-1}^{+}} u\right)<\varepsilon .
$$

Theorem 3.5. $\left(F_{p}\left(R^{n}\right), d_{p}\right)$ is separable. 
Proof. Since $\left(K\left(R^{n}\right), h\right)$ is separable, there exists a countable dense subclass $\mathcal{K}$ of $K\left(R^{n}\right)$.

Now let $\mathcal{F}$ be the family of fuzzy sets $v$ which for some positive $m$, there exist a finite unions $A_{1} \supset \cdots \supset A_{m}$ of sets in $\mathcal{K}$ and rational points $0<\alpha_{1} \leq \cdots \leq \alpha_{m-1}<1$ such that

$$
v(x)=\sum_{k=1}^{m-1} \alpha_{k} I_{A_{k} \backslash A_{k+1}}(x)+I_{A_{m}}(x),
$$

where $I_{A}$ denotes the indicator function of $A$.

Then it is obvious that $\mathcal{F}$ is countable subset of $F_{p}\left(R^{n}\right)$.

Now it suffices to prove that $\mathcal{F}$ is dense in $\left(F_{p}\left(R^{n}\right), d_{p}\right)$. Let $u \in F_{p}\left(R^{n}\right)$ and $\varepsilon>0$ be given. First we choose $0<\beta<1$ so that

$$
\int_{0}^{\beta}\left\|L_{\alpha} u\right\|^{p} d \alpha<(\varepsilon / 16)^{p}
$$

And then, by applying Lemma 3.4, we choose a partition $\beta=\beta_{0}<\cdots<\beta_{m}=1$ of $[\beta, 1]$ such that

$$
h\left(L_{\beta_{k}} u, L_{\beta_{k-1}^{+}} u\right)<\varepsilon / 8 \text { for all } k=1, \cdots, m .
$$

If we take $B_{k} \in \mathcal{K}, k=1,2, \cdots, m$ so that

$$
h\left(B_{k}, L_{\beta_{k}} u\right)<\varepsilon / 8 \text { for each } k \text {. }
$$

and let $A_{k}=\cup_{i=k}^{m} B_{i}$, then by lemma 3.3,

$$
h\left(L_{\beta_{k}} u, A_{k}\right)<\varepsilon / 8,
$$

and

$$
h\left(L_{\beta_{k-1}^{+}} u, A_{k}\right) \leq h\left(L_{\beta_{k-1}^{+}} u, L_{\beta_{k}} u\right)+h\left(L_{\beta_{k}} u, A_{k}\right)<\varepsilon / 4 .
$$

Let $\alpha_{m}=\beta_{m}=1$ and for each $k=1, \cdots, m-1$, we choose rational points $\alpha_{k}$ so that

$$
\beta_{k-1}<\alpha_{k} \leq \beta_{k}, h\left(L_{\alpha_{k}} u, L_{\beta_{k}} u\right)<\varepsilon / 8
$$

and

$$
\sum_{k=1}^{m} \int_{\alpha_{k}}^{\beta_{k}}\left(\left\|L_{\alpha} u\right\|+\left\|A_{1}\right\|\right)^{p} d \alpha<\varepsilon^{p} / 4 .
$$

Then

$$
h\left(L_{\alpha_{k}} u, A_{k}\right) \leq h\left(L_{\alpha_{k}} u, L_{\beta_{k}} u\right)+h\left(L_{\beta_{k}} u, A_{k}\right)<\varepsilon / 4 .
$$

Now if we define

$$
v(x)=\sum_{k=1}^{m-1} \alpha_{k} I_{A_{k} \backslash A_{k+1}}(x)+I_{A_{m}}(x),
$$

then

$$
L_{\alpha} v=\left\{\begin{array}{ccc}
A_{1} & \text { if } & 0<\alpha \leq \alpha_{1}, \\
A_{k} & \text { if } & \alpha_{k-1}<\alpha \leq \alpha_{k}, k=2, \cdots, n .
\end{array}\right.
$$

Since for $0<\alpha \leq \beta$,

$$
\begin{aligned}
h\left(L_{\alpha} u, L_{\alpha} v\right) & \leq h\left(L_{\alpha} u, L_{\beta^{+}} u\right)+h\left(L_{\beta^{+}} u, A_{1}\right) \\
& \leq 2\left\|L_{\alpha} u\right\|+\varepsilon / 4 \text { by }(2),
\end{aligned}
$$

we have

$$
\begin{aligned}
& \int_{0}^{\beta} h\left(L_{\alpha} u, L_{\alpha} v\right)^{p} d \alpha \\
\leq & 2^{p}\left[4^{p} \int_{0}^{\beta}\left\|L_{\alpha} u\right\|^{p} d \alpha+(\varepsilon / 4)^{p} \beta\right] \\
\leq & (\varepsilon / 2)^{p}(1+\beta) \text { by }(1) .
\end{aligned}
$$

And for $1 \leq k \leq m$

$$
\begin{aligned}
& \int_{\beta_{k-1}}^{\beta_{k}} h\left(L_{\alpha} u, L_{\alpha} v\right)^{p} d \alpha \\
&= \int_{\beta_{k-1}}^{\alpha_{k}} h\left(L_{\alpha} u, L_{\alpha} v\right)^{p} d \alpha+\int_{\alpha_{k}}^{\beta_{k}} h\left(L_{\alpha} u, L_{\alpha} v\right)^{p} d \alpha \\
& \leq \quad {\left[h\left(L_{\beta_{k-1}^{+}}^{+} u, A_{k}\right) \vee h\left(L_{\alpha_{k}} u, A_{k}\right)\right]^{p}\left(\alpha_{k}-\beta_{k-1}\right) } \\
&+\int_{\alpha_{k}}^{\beta_{k}}\left(\left\|L_{\alpha} u\right\|+\left\|A_{k}\right\|\right)^{p} d \alpha \\
& \leq \quad(\varepsilon / 4)^{p}\left(\beta_{k}-\beta_{k-1}\right)+\int_{\alpha_{k}}^{\beta_{k}}\left(\left\|L_{\alpha} u\right\|+\left\|A_{k}\right\|\right)^{p} d \alpha \\
& \text { by (2) and (4). }
\end{aligned}
$$

Therefore, we conclude that

$$
\begin{aligned}
& d_{p}^{p}(u, v) \\
= & \int_{0}^{\beta} h\left(L_{\alpha} u, A_{1}\right)^{p} d \alpha+\sum_{k=1}^{m} \int_{\beta_{k-1}}^{\beta_{k}} h\left(L_{\alpha} u, A_{k}\right)^{p} d \alpha \\
<\quad & (\varepsilon / 2)^{p}(1+\beta)+(\varepsilon / 4)^{p}(1-\beta) \\
& +\sum_{k=1}^{m} \int_{\alpha_{k}}^{\beta_{k}}\left(\left\|L_{\alpha} u\right\|+\left\|A_{1}\right\|\right)^{p} d \alpha \\
\leq \quad & 2 \varepsilon^{p} \quad \text { by (3). }
\end{aligned}
$$

This completes the proof.

We note that $\mathcal{F} \subset F_{\infty}\left(R^{n}\right)$ in the proof of Theorem 3.5. This means that $F_{p}\left(R^{n}\right)$ is the completion of $\left(F_{\infty}\left(R^{n}\right), d_{p}\right)$.

Remark. The results established in the above are valid even though $R^{n}$ is replaced by any real separable Banach space. 


\section{Reference}

[1] C. Degang, X. Xiaoping and Z. Liangkuan, "On the integrable noncompact fuzzy number space", Appl. Math.Lett. vol.21, pp.1260-1266, 2008.

[2] P. Diamond and P. Kloeden, Metric spaces of fuzzy sets: Theory and Applications, World Scientific, Singapore, 1994.

[3] G. H. Greco, "Sendograph metric and relatively compact sets of fuzzy sets", Fuzzy sets and Systems ,vol.157, pp.286-291,2006.

[4] G. H. Greco, "A characterization of relatively compactsets of fuzzy sets", Nonlinear Anal, vol.64, pp.518-529, 2006.

[5] G. H. Greco and M. P. Moschen, "Supremum metric and relatively compact sets of fuzzysets", Nonlinear Anal, vol.64, pp.1325-1335, 2006.

[6] S. Y. Joo and Y. K. Kim, "Topological properties on the space of fuzzy sets", Jour.Math.Anal.Appl. vol.246, pp.576-590, 2000.
[7] V. Kratschmer, "Some complete metrics on spaces of fuzzy sub sets", Fuzzy Sets and Systems, vol.130, pp.357-365, 2002.

[8] M. Ming, "Some notes on the characterization of compact sets in $\left(E^{n}, d_{p}\right)$ ", Fuzzy Sets and Systems, vol.56, pp.297-301, 1993.

[9] C. Wuand Z. Zhao, "Some notes on the characterization of compact sets with $L_{p}$ metric", Fuzzy Sets and Systems vol.159, pp.2104-2115, 2008.

[10] Z. Zhaoand $\mathrm{C}$. Wu, "The equivalence of convergence of sequences of fuzzy numbers and its applications to the characterization of compact sets", Information Sciences vol.179, pp.3018-3025, 2009.

\section{Yun Kyong Kim}

Professor of Dongshin University

Research Area: Fuzzy Probability Theory, Fuzzy Analysis and Related Fields

E-mail : ykkim@dsu.ac.kr 